\title{
Basic system of modelling (BSM): the concept, architecture and methodology*
}

\section{V.P. Il'in, V.S. Gladkih**}

Institute of Computational Mathematics and Mathematical Geophysics of the Siberian Branch of the RAS, Novosibirsk, Russian Federation

Novosibirsk State University, Novosibirsk, Russian Federation

The paper covers the architecture and main functional components of the basic system of modelling (BSM), as the integrated high-performance computational environment to solve interdisciplinary direct and inverse problems on the multi-processor computational system (MPS) with distributed and shared hierarchical memory. BSM subsystems support the stages of geometric and functional modelling, grid generation, high order approximations, fast algebraic solutions, etc. The scalable parallelism is provided by means of hybrid programming models: MPI, OpenMP, computational libraries for graphic accelerators and vectorization. The BSM main principles are discussed to have wide applications and long life cycle.

Keywords: Basic system of modelling; environment for high-performance computation; large-scale computational experiment, parallel algorithms, math model

Introduction. The present situation and world trends in the development of mathematical modeling have three main features. The first is the rapid growth of supercomputer capacities with the forthcoming appearance in the coming years of an exascale computing $\left(10^{18}\right.$ operations per second and up to $10^{9}$ computing units) provide enormous computationally-informative possibilities. This evolution is also included the complication of the architecture of multiprocessor computing systems with distributed and hierarchical shared memory, with the complex organization of multithreaded and vectorized operations, using various types of accelerators and cloud computing, without the effective use of which the overall high performance can not be ensured.

The second point is the sharply increased level of requirements for the completeness of the models under study that require solving interdisciplinary forward and backward initial-boundary value problems described by nonlinear systems of differential and integral equations in classical or generalized variational formulations with real data including complex configurations of multidimensional computational domains with multiply connected piecewise-smooth boundary surfaces, multiscale geometric details and contrasting material properties of media. A large-scale machine experiment to ensure effective predictive modeling involves the use of a hierarchy of physical and mathematical models to achieve, on the one hand, high resolution and the adequacy of numerical results, and on the other hand, the operative and visual acquisition of the basic properties of the objects under study.

\footnotetext{
* The research is supported by the grant of RSF Project No. 14-11-00485 and RFBR No 16-29-15122 ofi_m.

** E-mail: victor_gladkikh@lapasrv.sscc.ru, ilin@sscc.ru.
} 
The third important aspect of the current historical situation is the extremely active development of mathematics itself: theoretical, applied and computational, as well as programming technologies and artificial intelligence. Here we should note the following symbolic trends: the transition to algorithms that possess the laws of substance conservation (including those based on the Hamiltonian formalism) and high accuracy orders, fast methods for solving problems of computational algebra of extremely large dimension, scalable parallelism at various stages of the computational process, supporting high performance of computations by means of hybrid programming, automation of construction of algorithms for the purpose of increasing the productivity of programmers mathematicians.

The combined potential of mathematical modeling makes it, along with theoretical research and field experiments, the main tool for obtaining fundamental knowledge, as well as conducting research and development $(\mathrm{R} \& \mathrm{D})$ in all kinds of manufacturing industries. However, in order for these expectations to be fulfilled, it is necessary to carry out a huge amount of work on the creation of new generation software, which should bring the computing and information technologies to the level of professional tools for a wide range of users.

The traditional forms of the applied software are open source or commercial software packages aimed at solving a certain class of problems, among which we will mention only two popular developments - ANSYS [1] and Fenics [2]. There is also a large number of methodoriented libraries of programs (NETLIB [3], MKL [4] and many others), which are useful but auxiliary material for solving specific problems.

An alternative trend, manifested in recent decades, is the creation of integrated software environments open for participation in their development of various groups of developers of all kinds of applications. Examples of such projects are OpenFoam [5], DUNE (Distributed Unified Environment [6]) and MATLAB [7]. In ICMMGSB RAS, research has been carried out for a number of years on the development of the concept, architecture and methodology of the basic modeling system (BSM [8], [9]), aimed at coordinated support of all major technological stages of a large-scale computational experiment. The purpose of this work is to describe the overall structure, components of functional content and the principles of building BSM.

1. Functional filling of the basic stages of modelling. The concept of BSM is to create an integrated computing environment consisting of sufficiently autonomous components, or blocks, each of which provides an appropriate technological stage of modeling and interacts with the rest of the blocks through coherent data structures. The aggregate of these components forms the instrumental core of the BSM, the main purpose of which is assembly, or the formation, for each task, of the necessary configuration of programs (and if necessary, its implementation), designed to effectively implement a particular class of applications. And the computational session consists of sequential or cyclic execution of the following steps.

Geometric and functional modeling. The content of this stage consists in describing the given mathematical model of the task for the subsequent automated generation of the computational information model, which in future is represented by the source or executable program code. Typical geometric objects are subdomains, boundary surface segments, edges and vertices, and typical actions on them include shift, rotation, compression (stretching), and set of theoretic operations, including those involving a change in topology. In the field of analytic and differential 
geometry there are many beautiful results that still await their programmatic realization: here is the apparatus of the R-functions of the Kharkov school VL Rvachev [10], and differential or discrete forms on manifolds, and optimization of geometric structures [11] . On the other hand, a significant amount of geometric technologies are embedded in CAD products (CAD, SAE, CAM, PLM, [12]), which have a wide world distribution and with which FMC should naturally have close information agreement. In particular, the domestic project GERBARY has been chosen as such partner [13]. The result of geometric modeling is the geometric data structure (GDS) [14].

The functional objects of the mathematical model are the types and coefficients of the solved equations, the initial and boundary conditions and other data that together form the functional data structure (FDS), which, together with the GDS, reflects the entire formulation of the problem.

The construction of nets is an independent science-intensive problem, the solution of which is devoted to a large number of publications, special conferences and software products - public or commercial, for example, NETGEN [15] and GMESH [16]. The most common are adaptive grids that adjust to the geometric features of the computational domain and the differential properties of the desired solution. In practical problems, such grids are unstructured, in which the properties of the neighborhood of nodes can only be indicated by an enumeration. [17] describes the main characteristics of the DELAUNAY library, which is an integrated environment for the generation of quasistructured grids, in which the grid computational domain can consist of subareas with its different types of subnets. The functional content of the library includes both original algorithms and the coordinated use of external grid generators. The result of the DELAUNAY work is the grid (mesh) data structure (MDS), which, taking into account the GDS and the FDS, completely determines the discretized formulation of the problem. We note such an important technological aspect that for effective scalable parallelization based on domain decomposition at this stage it is necessary to conduct the formation of grid subdomains and corresponding distributed on the basis of the MDS processors, in accordance with the a priori estimate of the laboriousness of computations in each subregion.

Approximation of the initial equations in the theoretical plan makes the most responsible stage of modeling. The most common approaches here are finite-volume methods (FVM [18]), finite elements (FEM [19]) and Galerkin discontinuous algorithms of various accuracy orders that all have a universal technological base - parallel element-by-element principles for computing local matrices and assembling global matrices in typical sparse formats. The resulting algebraic data structures (ADS) for large tasks are generated by distributed grid sub-regions and by the corresponding processors. In BSM, the entire implementation of approximation methodologies is carried out by the library CHEBYSHEV [20].

The solution of algebraic problems is the most resource-intensive computational stage realized in BSM with the help of the KRYLOV library [21]. Modern approaches to the implementation of large sparse SLAE are based on two-level preconditioned iterative methods for the balanced decomposition of regions in Krylov subspaces, accelerated by means of coarse-grained correction, least squares, aggregation, deflation, and low-rank approximation of the original matrices, and by optimizing the intersection parameters of subdomains and interface conditions on their internal boundaries. Scalable parallelism is achieved by means of hybrid programming, using 
MPI, OpenMP, graphics accelerators and vectorization of computations, as well as using data buffering to minimize communication losses between sub-regions. Effective realization of algorithms based on ADS, represented by compressed sparse formats such as CSR (Compressed Sparse Row) with active use of functions of the Sparse BLAS library. The architecture of the KRYLOV library supports interaction with external software products such as PETSc.

General principles and technical requirements for the construction of BSM. The conceptual principles of building BSM proceed from the definition of it as an open method-oriented integrated computing and information environment for solving a wide class of mathematical modeling problems oriented to a long life cycle with active application by various groups of developers and end users. Based on this general formulation, the following technical requirements can be defined, which should ensure the success of the program project under consideration.

- Flexible extensibility of the model of solved interdisciplinary problems, as well as methods for solving direct and inverse problems, without software restrictions on the number of degrees of freedom and the number of computing processors and cores used.

- Adaptability to the evolution of computer architectures. Component technologies for ensuring the consistency of internal and external intermodule interfaces.

- Flexible extensible data structures, consistent with existing common formats and supporting the re-use of external software products.

- Multilingual and cross-platform software functional content, openness to coordinated participation in the project of various groups of developers.

- High performance code with scalable parallelism based on hybrid programming on heterogeneous AIM with distributed and hierarchical shared memory.

- Presence of various user interfaces with orientation to wide application in various industrial spheres.

Conclusion. Principles and requirements for BSM were formed from the experience of implementing new mathematical techniques in the form of a program for application in practical tasks. The increasing demands on the detail of mathematical models and the speed of their implementation in the program code led to the fact that the increase in labor efficiency turned out to be in the focus of world attention, and the creation on the basis of the accumulated experience of tools is an important task of this century. We would like to especially note that one of the factors for the further success of the project is the formation of a community of developers and users, which ensures further accumulation of experience and acceleration of development environment development.

\section{References}

1. ANSYS. URL: www.ansys.com (reference date: July 31, 2017)

2. FEniCS. URL: http://fenicsproject.org/ (reference date: July 31, 2017)

3. Netlib. URL: http://www.netlib.org/ (reference date: July 31, 2017)

4. Intel@. Mathematical Kernel Library. URL: http://software.intel.com/en-us/intel-mkl (reference date: July 31, 2017)

5. OpenFOAM. URL: http://www.openfoam.com (reference date: July 31, 2017)

6. DUNE. http: //www, dune-project.org (reference date: July 31, 2017) 
7. MATLAB. URL: https://www.mathworks.com/products/matlab.html (reference date: July $31,2017)$

8. Il'in, V.P. On the performance and intelligence of supercomputer modeling. / Il'in V.P., Skopin I.N. // Programming. - 2016. - No. 1. - pp. 10-25.

9. Il'in, V.P. Fundamental questions of mathematical modeling. / Il'in V.P. // Bulletin of the Russian Academy of Sciences. - 2016. - Vol. 86. - No. 4. - pp. 26-36.

10. Kravchenko, V.F. Algebra of logic, atomic functions and wavelets in physical applications / Kravchenko V.F., Rvachev V.L. // Moscow: Fizmatlit, 2006.

11.Delfour, M. Metrics, Analysis, Differential Calculus, and Optimization. / Delfour M., Zolesio J. Shapes and Geometries. // Philadelphia: SIAM - 2011.

12. Ushakov, D.M. Introduction to the mathematical foundations of CAD. / Ushakov D.M. // Novosibirsk: Publishing house of CJSC LEDAS. - 2008.

13. The Herbarium. URL: http://tflex.ru/about/publications/detail/index.php?ID=3846 (reference date: July 31, 2017)

14. Golubeva, L.A. On software technologies in geometric aspects of mathematical modeling / Golubeva L.A., Ilyin V.P., Kozyrev A.N. // Bulletin of NSU. Series «Information Technologies». - 2012. - Vol. 10. - pp. 25-33.

15. Schoberl J. Netgen-an advancing front 2D / 3D-mesh generator based on abstract rules / Schoberl J. // Computing and Visualization in Science. 1997. - Vol. 1. - pp. 41-52.

16. GMESH. URL: http://gmsh.info/ (reference date: July 31, 2017)

17. Il'in, V.P. DELAUNAY: technological environment of grid generation / Il'in V.P. // SIBLIM. - 2013. - Vol.16. - pp. 83-97.

18. Il'in, V.P. Methods of finite differences and finite volumes for elliptic equations. / Il'in V.P. // Novosibirsk: Publishing house of the IVMMG SB RAS. - 2001.

19. Il'in V.P. Methods and technologies of finite elements. / Il'in V.P. // Novosibirsk: Publishing house of the IVMMG SB RAS. - 2007.

20. Butyugin, D.S. Chebyshev: principles of automating the construction of algorithms in an integrated environment for grid approximations of initial-boundary value problems. / Butyugin D.S., Il'in V.P. // Proceedings of the International Conference PCT-2014. Chelyabinsk: Publishing House of SUSU. - 2014. - pp. 42-50.

21. Butyugin, D.S. Functionality and technology of algebraic solvers in the Krylov library. / Butyugin DS, Guryeva Ya.L., Il'in V.P. and others // Herald of SUSU. Series «Computational Mathematics and Informatics». - 2013. - Vol. 2. - pp. 92-105.

22. Il'in, V.P. On the problems of solving large SLAE. / Il'in V.P. // Zapiski Nauchnykh Seminarov POMI RAN - 2015 - pp. 112-127.

23. Il'in, V.P. On the numerical solution of direct and inverse problems of electromagnetic electrical prospecting / Il'in V.P. // Siberian Journal of Computational Mathematics - 2003 - Vol. 6 - pp. 381-391

24. Il'in, V.P. About component technologies of high-performance mathematical modeling. / Ilyin V.P. // Proceedings of the Intern. Conf. PCT-2015. Ekaterinburg: Ufa, IMM UrB RAS - 2015 - pp. 166-171. 
Authors:

Il'in Valery Pavlovich, Doctor of Science in Physics and Maths, Professor, Institute of Computational Mathematics and Mathematical Geophysics of the Siberian Branch of the RAS, Novosibirsk, Russian Federation (6 Prospekt Academician Lavrentyev, Novosibirsk, Russian Federation)

Gladkih Viktor Sergeevich, engineer of the 1st category, Institute of Computational Mathematics and Mathematical Geophysics of the Siberian Branch of the RAS, Novosibirsk, Russian Federation (6 Prospekt Academician Lavrentyev, Novosibirsk, Russian Federation) 
УДК 004.421

Базовая система моделирования (БСМ): концепция, архитектура и методология *

\section{В.П. Ильин, В.С. Гладких **}

Институт вычислительной математики и математической геофизики СО РАН, Новосибирский Государственный Университет, Новосибирск, Российская Федерация

Рассматриваются архитектура и основные компоненты базовой системы моделирования (БСМ), являющейся высокопроизводительной вычислительной средой разработки для решения прямых и обратных задач на много-процессорных системах (MBC) с распределенной и иерархической общей памятью. Модули системы БСМ содержат средства геометрического и функционального моделирования, генерации сетки, аппроксимаций высокого порядка, быстрых алгебраических решателей и т.д. Эффективное распараллеливание достигается за счет использования гибридных программных средств: MPI, OpenMP; векторизации кода, вычислительных библиотек для графических ускорителей.

Ключевые слова: Базовая система моделирования; среда разработки высокопроизводительных математических приложений; поддержка крупномасштабного вычислительного эксперимента.

\section{Авторы:}

Ильин Валерий Павлович, доктор физико-математических наук, профессор, Институт вычислительной математики и математической геофизики Сибирского отделения РАН (ИВМиМГ СО РАН) (630090, Новосибирск, Россия, Проспект академика Лаврентьева, 6)

Гладких Виктор Сергеевич, инженер 1 категории, Институт вычислительной математики и математической геофизики Сибирского отделения РАН (ИВМиМГ СО РАН) (630090, Новосибирск, Россия, Проспект академика Лаврентьева, 6)

\footnotetext{
* Работа поддержана грантами РНФ №14-11-00485П и РФФИ №16-29-15122 офи_м.

** E-mail: victor_gladkikh@lapasrv.sscc.ru, ilin@sscc.ru.
} 